\title{
HMBOX1 attenuates LPS-induced periodontal ligament stem cell injury by inhibiting CXCL10 expression through the NF-кB signaling pathway
}

\author{
MINYUAN NIE ${ }^{1}$, HENG LI $^{2}$, PUHE LIU $^{3}$ and PING DANG ${ }^{4}$ \\ ${ }^{1}$ Department of Stomatology, PLA Strategic Support Force Characteristic Medical Center, Beijing 100101; \\ ${ }^{2}$ Department of Paediatric Dentistry, Nanjing Stomatological Hospital, Medical School of Nanjing University, Nanjing, \\ Jiangsu 210008; ${ }^{3}$ Department of Stomatology, The First Dental Hospital, Wuhai, Inner Mongolia Autonomous Region 016000; \\ ${ }^{4}$ Department of Stomatology, Amcare Women's and Children's Hospital, Beijing 100016, P.R. China
}

Received April 20, 2021; Accepted July 21, 2021

DOI: $10.3892 /$ etm.2022.11148

\begin{abstract}
Homeobox containing 1 (HMBOX1) is a member of the homeobox transcription factor family that has been reported to serve an important role in numerous biological processes. The present study aimed to determine the role of HMBOX1 in the pathogenesis of periodontitis. Human periodontal ligament stem cells (hPDLSCs) were treated with liposaccharide (LPS) and transfected with a HMBOX1 overexpression (Ov-HMBOX1) plasmid or small interfering (si)-C-X-C motif chemokine ligand 10 (CXCL10) plasmids The effects of Ov-HMBOX1 on cell proliferation, inflammation and apoptosis were subsequently investigated using Cell Counting Kit-8, ELISA for analysis of IL-6, TNF- $\alpha$ and IL-1 $\beta$ levels, TUNEL and western blotting assays for analysis of Bcl-2, Bax, cleaved caspase-3 and caspase-3 levels, respectively. Furthermore, the potential effects of HMBOX1 on the mRNA and protein levels of CXCL10 and the NF- $\kappa$ B signaling pathway were investigated by using reverse transcription-quantitative PCR and western blotting. Finally, the physiological processes of lipopolysaccharide (LPS)-induced hPDLSCs overexpressing HMBOX1 were assessed following treatment with phorbol 12-myristate 13-acetate (PMA), a NF- $\kappa$ B agonist. The results revealed that Ov-HMBOX1 transfection promoted proliferation whilst alleviating inflammation and apoptosis in LPS-induced hPDLSCs. Ov-HMBOX1 reduced the expression of CXCL10 by suppressing the NF- $\kappa$ B signaling pathway. PMA treatment inhibited the proliferation of LPS-induced hPDLSCs transfected with Ov-HMBOX1, which was reversed by transfection with si-CXCL10. In conclusion, results of the
\end{abstract}

Correspondence to: Dr Ping Dang, Department of Stomatology, Amcare Women's and Children's Hospital, 9 Fangyuan Xilu, Chaoyang, Beijing 100016, P.R. China

E-mail: dangpingd@126.com

Key words: homeobox containing 1, periodontitis, C-X-C motif chemokine ligand $10, \mathrm{NF}-\mathrm{\kappa B}$ present study provided evidence that HMBOX1 can attenuate LPS-induced hPDLSC injury by downregulating CXCL10 expression via the $\mathrm{NF}-\kappa \mathrm{B}$ signaling pathway, which may provide a novel insight into the development of potentially novel treatment strategies for periodontitis.

\section{Introduction}

Periodontitis is one of the most common chronic inflammatory diseases and is characterized by the progressive destruction of the supporting tissues surrounding the tooth, eventually leading to tooth loss (1-3). Due to the lack of clear symptoms, the majority of patients typically miss the early intervention period of this disease, meaning that extensive damage has already been caused to the entire dentition on presentation (4). A review has previously revealed an association between periodontitis and diabetes complications in the human body (5). Periodontitis is traditionally treated using non-surgical methods, which mainly involve the subgingival eradication of bacterial deposits on the dental surface (6). However, deeper understanding on the etiopathogenesis of the disease is required to develop novelly effective treatment options (1).

Homeobox containing 1 (HMBOX1) is a member of the homeobox transcription factor family that has been identified to serve a role in a large number of biological processes including proliferation, inflammation and apoptosis (7-10). A previous genomic and proteomic analysis found that the expression levels of HMBOX1 were downregulated in gingival tissues from patients with periodontitis compared with a control group without periodontitis, which identified a potential association between HMBOX1 expression levels and the pathogenesis of periodontitis (11). In addition, mice deficient in HMBOX1 exhibited enhanced levels of apoptosis in vascular endothelial cells following challenge with lipopolysaccharide (LPS), which promoted the inflammatory response in a mouse model of acute lung injury $(10,12)$. However, to the best of our knowledge, no studies to date have determined the specific role of HMBOX1 in the pathogenesis of periodontitis.

CXCL10 was first identified in 1985 and has been found to be regulated by various cytokines, such as IFN- $\gamma$ and 
TNF (13-16). It has been previously reported that the significantly upregulated expression of CXCL10 in patients with periodontitis can serve as a biomarker of periodontitis (17). In addition, the NF- $\mathrm{NB}$ signaling pathway was found to upregulate the expression of CXCL10 in COMM domain-containing protein 7-treated hepatocellular carcinoma cells (18). Aldo-keto reductase family 1 member $\mathrm{C} 2$ was also discovered to exert potent anti-inflammatory and antioxidant effects by inhibiting $\mathrm{NF}-\kappa \mathrm{B}$ activity downstream of HMBOX1 activation in an atherosclerosis model of LPS-induced EA.hy926 cells (9).

Therefore, it was hypothesized in the present study that HMBOX1 may alleviate LPS-induced human periodontal ligament stem cell (hPDLSC) injury by downregulating CXCL10 expression via the $\mathrm{NF}-\kappa \mathrm{B}$ signaling pathway.

\section{Materials and methods}

Cell lines and culture.Human periodontal stemcells (hPDLSCs) were purchased from YBIO (cat. no. YB-Cell-H002) and cultured in DMEM (Gibco; Thermo Fisher Scientific, Inc.) supplemented with $10 \%$ FBS (Gibco; Thermo Fisher Scientific, Inc.) in a humidified incubator with $5 \% \mathrm{CO}_{2}$ at $37^{\circ} \mathrm{C}$. Cells were treated with or without $1 \mu \mathrm{g} / \mathrm{ml}$ LPS (cat. no. L8880; Beijing Solarbio Science \& Technology Co., Ltd.) for 12 or $24 \mathrm{~h}$ at $37^{\circ} \mathrm{C}$ to construct the in vitro periodontitis model. The NF- $\kappa \mathrm{B}$ activator phorbol 12-myristate 13-acetate (PMA) (19) $(20 \mathrm{ng} / \mathrm{ml}$; Sigma-Aldrich; Merck KGaA) was used to pre-treat hPDLSCs for $30 \mathrm{~min}$ at $37^{\circ} \mathrm{C}$ on hPDLSCs before LPS treatment and then transfected with the HMBOX1 overexpression (Ov-HMBOX1, pEGFP-N1; vector) plasmid or its control plasmids (Ov-NC, empty plasmids) for $24 \mathrm{~h}$.

Cell transfection. Ov-HMBOX1 $(1 \mu \mathrm{g})$ and its negative control (Ov-NC; empty plasmids. $1 \mu \mathrm{g}$ ), short interfering RNA (siRNA/si) for CXCL10 (30 pmol; si-CXCL10-1, 5'-GGU UAAUAA AGUAAUUAUAAC-3'; si-CXCL10-2, 5'-CGU GUUGAGAUCAUUGCUACA-3') and si-NC (30 pmol; 5'-GAUAAUUAUGGAUAAUAAUAC-3') were purchased from Shanghai GenePharma Co., Ltd. Transfection was performed using Lipofectamine ${ }^{\circledR} 3000$ reagent (Invitrogen; Thermo Fisher Scientific, Inc.) according to the manufacturer's protocol. Transfected cells were cultured for $24 \mathrm{~h}$ at $37^{\circ} \mathrm{C}$ prior for use in subsequent experiments.

Reverse transcription-quantitative PCR (RT-qPCR). Total RNA was extracted from hPDLSCs using TRIzol ${ }^{\circledR}$ reagent (Invitrogen; Thermo Fisher Scientific, Inc.). Total RNA $(2 \mu \mathrm{g})$ was reverse-transcribed into cDNA using a PrimeScript ${ }^{\mathrm{TM}} \mathrm{RT}$ kit (Takara Bio, Inc.) under a condition of $37^{\circ} \mathrm{C}$ for $1 \mathrm{~h}$ and $72^{\circ} \mathrm{C}$ for $10 \mathrm{~min}$. qPCR was subsequently performed using a SYBR ${ }^{\circledR}$ Premix Ex Taq ${ }^{\mathrm{TM}}$ (cat. no. RR420A; Takara Bio, Inc.) on a LightCycler 480 PCR system (Roche Diagnostics) according to the manufacturer's protocol. The following thermocycling conditions were used for the qPCR: Initial denaturation at $95^{\circ} \mathrm{C}$ for $2 \mathrm{~min}$; followed by 40 cycles of $95^{\circ} \mathrm{C}$ for $20 \mathrm{sec}$, $60^{\circ} \mathrm{C}$ for $20 \mathrm{sec}$ and $72^{\circ} \mathrm{C}$ for $20 \mathrm{sec}$, followed by a final step at $78^{\circ} \mathrm{C}$ for $5 \mathrm{~min}$. The primers sequences are as following: HMBOX1 forward, 5'-CTTCAGCGACTTCGGCGTA-3' and reverse, 5'-ATCATAACTGTTGCTAGGTG ACG-3'; CXCL10 forward, 5'-GTGGCATTCAAGGAGTACCTC-3' and reverse,
5'-TGATGGCCTTCGATTCTGGATT-3' and GAPDH forward, 5'-GGAGCGAGATCCCTCCAAAAT-3' and reverse, 5'-GGCTGTTGTCATACTTCTCATGG-3' Relative mRNA expression levels were quantified using the $2^{-\Delta \Delta \mathrm{Cq}}$ method and normalized to GAPDH expression (20).

Western blotting. Total protein was extracted from hPDLSCs using RIPA lysis buffer (Beyotime Institute of Biotechnology). Total protein was quantified using a bicinchoninic acid assay kit (ProteinTech Group, Inc.) and $50 \mu \mathrm{g}$ protein/lane was separated by $12 \%$ SDS-PAGE. The separated proteins were subsequently transferred onto nitrocellulose membranes and non-specific binding was blocked with $5 \%$ skimmed milk diluted in TBS buffer for $3 \mathrm{~h}$ at room temperature. The membranes were then incubated overnight at $4^{\circ} \mathrm{C}$ with specific primary antibodies against HMBOX1 (cat. no. 16123-1-AP; 1:1,000; ProteinTech Group,Inc.), CXCL10 (cat.no. 10937-1-AP; 1:1,000; Proteintech Group, Inc.), Ki67 (cat. no. ab16667; 1:1,000; Abcam), proliferating cell nuclear antigen (PCNA; cat. no. ab92552; 1:1,000); IL-6 (cat. no. ab233551; 1:1,000; Abcam), TNF- $\alpha$ (cat. no. ab183218; 1:1,000; Abcam), IL-1 $\beta$ (cat. no. ab216995; 1:1,000; Abcam), Bcl-2 (cat. no. ab32124; 1:1,000; Abcam), Bax (cat. no. ab32503; 1:1,000; Abcam), cleaved caspase-3 (cat. no. ab2302; 1:500; Abcam), caspase-3 (cat. no. ab184787; 1:2,000; Abcam), phosphorylated (p-) p65 (cat. no. ab76302, 1:1,000; Abcam), p65 (cat. no. ab32536; 1:1,000; Abcam), ІкB $\alpha$ (cat. no. sc-1643; 1:1,000; Santa Cruz Biotechnology, Inc.) p-IкB $\alpha$ (cat. no. sc-101713; 1:500; Santa Cruz Biotechnology, Inc.) and GAPDH (cat. no. ab9485; 1:1,000; Abcam). Following the primary antibody incubation, the membranes were washed three times with TBS-Tween-20 (0.05\% Tween-20) for $10 \mathrm{~min}$ each time and incubated with an HRP-conjugated secondary antibody (cat. no. ab7090; 1:10,000; Abcam) for $45 \mathrm{~min}$ at room temperature. Protein bands were visualized using Immun-Star HRP Chemiluminescent Substrate kit (cat. no. 1705040) on a detector (both from Bio-Rad Laboratories, Inc.). The grey value was quantified with ImageJ software 1.46r (National Institutes of Health).

Cell Counting Kit-8 (CCK-8) assay. Cells were seeded into 96-well plates at a density of $2 \times 10^{4}$ cells/well and cultured for $24 \mathrm{~h}$ at $37^{\circ} \mathrm{C}$, after which $10 \mu \mathrm{l} C \mathrm{CK}-8$ reagent (Dojindo Molecular Laboratories, Inc.) was added to each well and incubated for a further $4 \mathrm{~h}$ at $37^{\circ} \mathrm{C}$. The absorbance was measured at a wavelength of $450 \mathrm{~nm}$ using a microplate reader.

ELISA. The cell supernatant of transfected hPDLSCs was collected and the levels of TNF- $\alpha$ (cat. no. EK0525), IL-6 (cat. no. EK0410) andIL-1 $\beta$ (cat. no. EK0392) were determined using their corresponding ELISA kits (Wuhan Boster Biological Technology Ltd.) according to the manufacturer's protocols.

TUNEL assay. Apoptosis was conducted using an In Situ Cell Death Detection kit (Roche Diagnostics) according to the manufacturer's protocol. The cells were fixed with $4 \%$ paraformaldehyde for $30 \mathrm{~min}$ at room temperature, followed by the permeation of $0.2 \%$ Triton $\mathrm{X}-100$ for $8 \mathrm{~min}$ at room temperature. TUNEL solution was dropped into sections for incubation for $60 \mathrm{~min}$ at $37^{\circ} \mathrm{C}$, which were then washed 

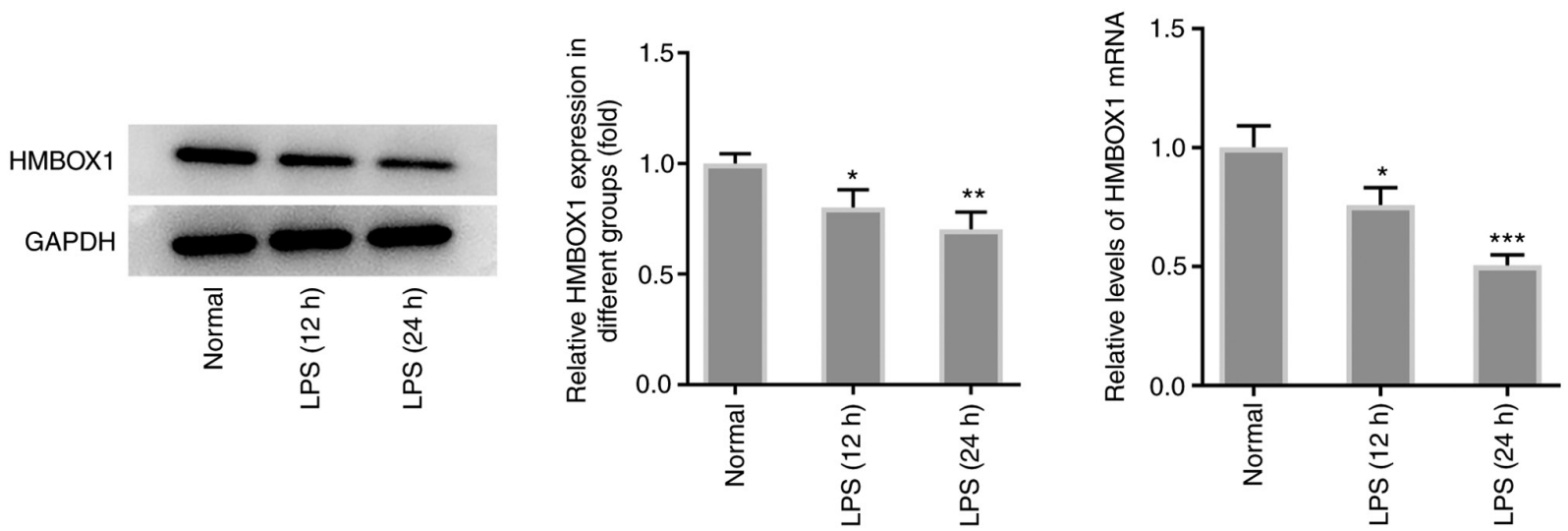

Figure 1. Expression levels of HMBOX1 in LPS-induced hPDLSCs. Protein and mRNA expression of HMBOX1 in LPS-induced hPDLSCs as measured by western blotting and reverse transcription-quantitative PCR, respectively. hPDLSCs, human periodontal ligament stem cells; HMBOX1, Homeobox containing 1 ; LPS, lipopolysaccharide. ${ }^{*} \mathrm{P}<0.05,{ }^{* * *} \mathrm{P}<0.01,{ }^{* * * *} \mathrm{P}<0.001$ vs. normal.

with PBS for three times, each for 5 min and the nuclei were stained using DAPI for $8 \mathrm{~min}(0.4 \mu \mathrm{g} / \mathrm{m})$ in the dark. Apoptotic cells in four randomly selected views were visualized using an inverted fluorescence microscope (IX73; Olympus Corporation; magnification, $\mathrm{x} 200)$. The apoptotic index (\%) was calculated using the following formula: Apoptotic cells (green fluorescence)/total cells (blue fluorescence) x100\%.

Statistical analysis. Statistical analysis was performed using GraphPad Prism version 6.0 software (GraphPad Software, Inc.) and data are presented as the mean \pm SD. Statistical differences between $\geq 3$ groups were determined using one-way ANOVA followed by Tukey's test. All experiments were repeated at least three times. $\mathrm{P}<0.05$ was considered to indicate a statistically significant difference.

\section{Results}

Overexpression of HMBOXI promotes the proliferation of $L P S$-induced hPDLSCs. To determine the role of HMBOX1 in the pathogenesis of periodontitis, the expression levels of HMBOX1 were first measured in LPS-treated hPDLSCs. As shown in Fig. 1, exposure of hPDLSCs to LPS for 12 or $24 \mathrm{~h}$ significantly downregulated the mRNA and protein expression levels of HMBOX1 compared with those in hPDLSCs without LPS treatment.

Subsequently, transfection of hPDLSCs with Ov-HMBOX1 significantly increased the expression of HMBOX1 compared with that in the control and Ov-NC groups (Fig.2A).Furthermore, results from the CCK- 8 and western blot analyses demonstrated that LPS exposure significantly reduced the viability of hPDLSCs and expression levels of the proliferation-related proteins Ki-67 and PCNA (Fig. 2B. By contrast, Ov-HMBOX1 significantly reversed all aforementioned effects on cell viability and Ki-67 and PCNA expression (Fig. 2B and C). These results suggest that HMBOX1 overexpression can restore the proliferation of LPS-induced hPDLSCs.

Overexpression of HMBOX1 inhibits inflammation and apoptosis in LPS-induced hPDLSCs. Since periodontitis is one of the most prevalent inflammatory diseases (21), the effects of
HMBOX1 on the inflammation and apoptosis of LPS-induced hPDLSCs were analyzed. LPS stimulation significantly increased the production and expression of inflammatory cytokines IL-6, TNF- $\alpha$ and IL-1 $\beta$, which was significantly reversed by Ov-HMBOX1 transfection (Fig. 3A and B). LPS stimulation also significantly increased the apoptosis of hPDLSCs, whilst significantly downregulating the expression levels of the anti-apoptotic proteins Bcl-2. However, expression of the proapoptotic proteins Bax and cleaved caspase-3 were significantly increased y LPS treatment (Fig. 3C and D). Conversely, these observations aforementioned were significantly reversed after these LPS-induced hPDLSCs were transfected with Ov-HMBOX1 (Fig. 3C and D). These findings suggest that HMBOX1 overexpression can inhibit the inflammation and apoptosis of LPS-induced hPDLSCs.

HMBOX1 modulates the expression of CXCL10 by regulating the $N F-\kappa B$ signaling pathway. To investigate the effects of HMBOX1 on CXCL10 expression and the NF- $\kappa B$ signaling pathway in LPS-induced hPDLSCs, western blotting and RT-qPCR were used to measure CXCL10 expression levels following the overexpression of HMBOX1. The results revealed that Ov-HMBOX1 transfection significantly downregulated the expression levels of CXCL10 compared with those in cells transfected with Ov-NC (Fig. 4A). Furthermore, LPS-induced phosphorylation of p65 and I $\mathrm{B} \alpha$ was significantly abrogated by Ov-HMBOX1 transfection (Fig. 4B), suggesting a possible association between HMBOX1 and the NF- $\kappa \mathrm{B}$ signaling pathway. Therefore, a NF- $\kappa \mathrm{B}$ agonist, PMA, was used to assess the potential effects of NF- $\kappa B$ on the regulation of CXCL10 expression. The significant suppressive effects of Ov-HMBOX1 on the expression of CXCL10 were significantly reversed by PMA (Fig. 4C). These findings suggest that HMBOX1 may modulate the expression of CXCL10 by regulating the NF- $\mathrm{BB}$ signaling pathway.

HMBOX1 enhances the proliferation whilst inhibiting the inflammation and apoptosis of LPS-induced hPDLSCs through the $N F-\kappa B / C X C L 10$ axis. Based on the aforementioned findings, it was hypothesized that HMBOX1 


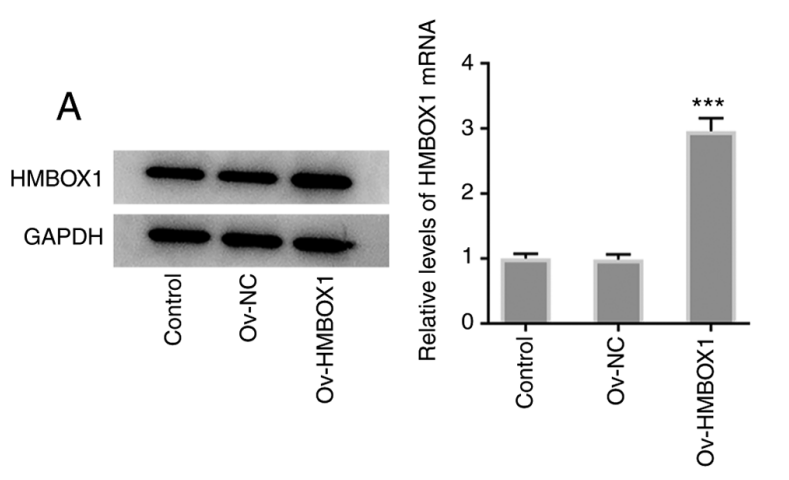

C

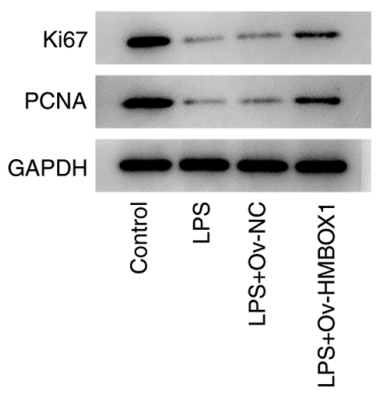

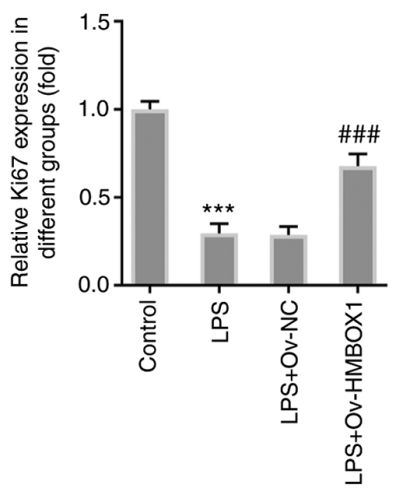

B

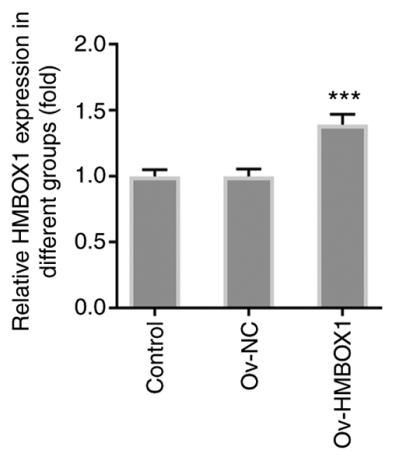

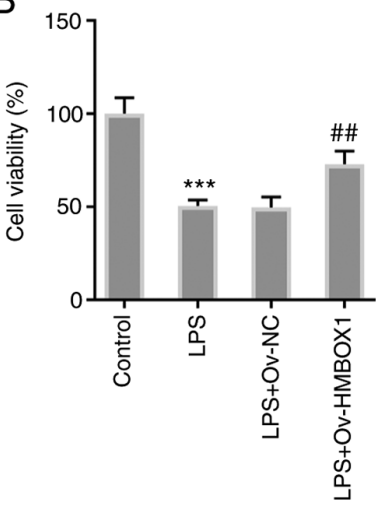

Figure 2. Overexpression of HMBOX1 promotes the proliferation of LPS-induced hPDLSCs. (A) HMBOX1 expression, ${ }^{* * *} \mathrm{P}<0.001$ vs. Ov-NC. (B) cell viability and $(\mathrm{C})$ the expression of proliferation-related proteins Ki-67 and PCNA were measured after LPS-induced hPDLSCs were transfected with the HMBOX1 overexpression plasmid. ${ }^{* * *} \mathrm{P}<0.001$ vs. control; ${ }^{\# \#} \mathrm{P}<0.01$, ${ }^{\# \# t} \mathrm{P}<0.001$ vs. LPS+Ov-NC. hPDLSCs, human periodontal ligament stem cells; HMBOX1, Homeobox containing 1; LPS, lipopolysaccharide; PCNA, proliferating cell nuclear antigen; Ov, overexpression; NC, negative control.

may influence the physiology of LPS-induced hPDLSCs through the $\mathrm{NF}-\kappa \mathrm{B} / \mathrm{CXCL} 10$ signaling axis. The expression of CXCL10 was knocked down using si-CXCL10-1 and si-CXCL10-2. si-CXCL10-2 was selected for use in subsequent experiments as it was able to significantly downregulate CXCL10 expression, with a markedly greater magnitude compared with that mediated by si-CXCL10-1 (Fig. 5A). PMA treatment inhibited the proliferation of hPDLSCs in LPS+Ov-HMBOX1+PMA+si-NC group compared with LPS+Ov-HMBOX1+si-NC group, which was significantly abolished by co-transfection with si-CXCL10 (Fig. 5B). Furthermore, the protein levels of proliferation markers, Ki67 and PCNA, were significantly decreased by PMA treatment compared with that in the co-treatment of LPS and Ov-HMBOX1 transfection, but were able to be markedly reversed by CXCL10 silencing (Fig. 5C).

The inflammation and apoptosis of hPDLSCs in LPS+Ov-HMBOX1+PMA+si-NC group were enhanced by PMA as relative to $\mathrm{LPS}+\mathrm{Ov}-\mathrm{HMBOX} 1$ group, as evidenced by the increased production and secretion of inflammatory cytokines IL-6, TNF- $\alpha$ and IL-1 $\beta$ (Fig. 6A and B) and the increased apoptotic rate of these cells. In addition, increased expression levels of Bax and cleaved caspase-3, and a decrease in Bcl-2 expression were observed after addition of PMA compared with the LPS+Ov-HMBOX1 group (Fig. 6C and D). By contrast, all of these effects aforementioned were reversed following co-transfection with si-CXCL10 (Fig. 6). Taken together, these data suggest that HMBOX1 may enhance the proliferation and inhibit the inflammation and apoptosis of
LPS-induced hPDLSCs through the NF- $\kappa \mathrm{B} / \mathrm{CXCL10}$ signaling axis.

\section{Discussion}

The homeobox family of protein contains $\sim 300$ gene members, most of which have been implicated in the regulation of embryonic development (22). In particular, HMBOX1 is ubiquitously expressed in human tissues and has been identified to be a novel transcription factor (6). HMBOX1 consists of a homeobox domain in the N-terminal region and an hepatocyte nuclear factor 1 domain at the C-terminus (7). In the present study, the expression levels of HMBOX1 were found to be downregulated in LPS-induced hPDLSCs. Therefore, the potential effects of HMBOX1 on the physiology of LPS-treated hPDLSCs were investigated by constructing an Ov-HMBOX1 plasmid and transfecting it into hPDLSCs. The cytotoxicity of LPS-induced hPDLSCs was mitigated following Ov-HMBOX1 transfection, which was accompanied by enhanced cell proliferation. In addition, the inflammation and apoptosis of LPS-induced hPDLSCs were alleviated following Ov-HMBOX1 transfection. The present study found also that increased HMBOX1 inhibited NF- $\mathrm{NB}$ activation in hPDLSCs in response to LPS. In addition to the potential involvement of $\mathrm{NF}-\kappa \mathrm{B}$ in the regulatory role of $\mathrm{HMBOX} 1$ in response to LPS, a previous study also showed that 5'AMP-activated protein kinase mediates the regulation of $\mathrm{HMBOX} 1$, which is associated with inflammation after EA.hy926 cells were subjected to LPS stimulation (9). Additionally, HMBOX1 

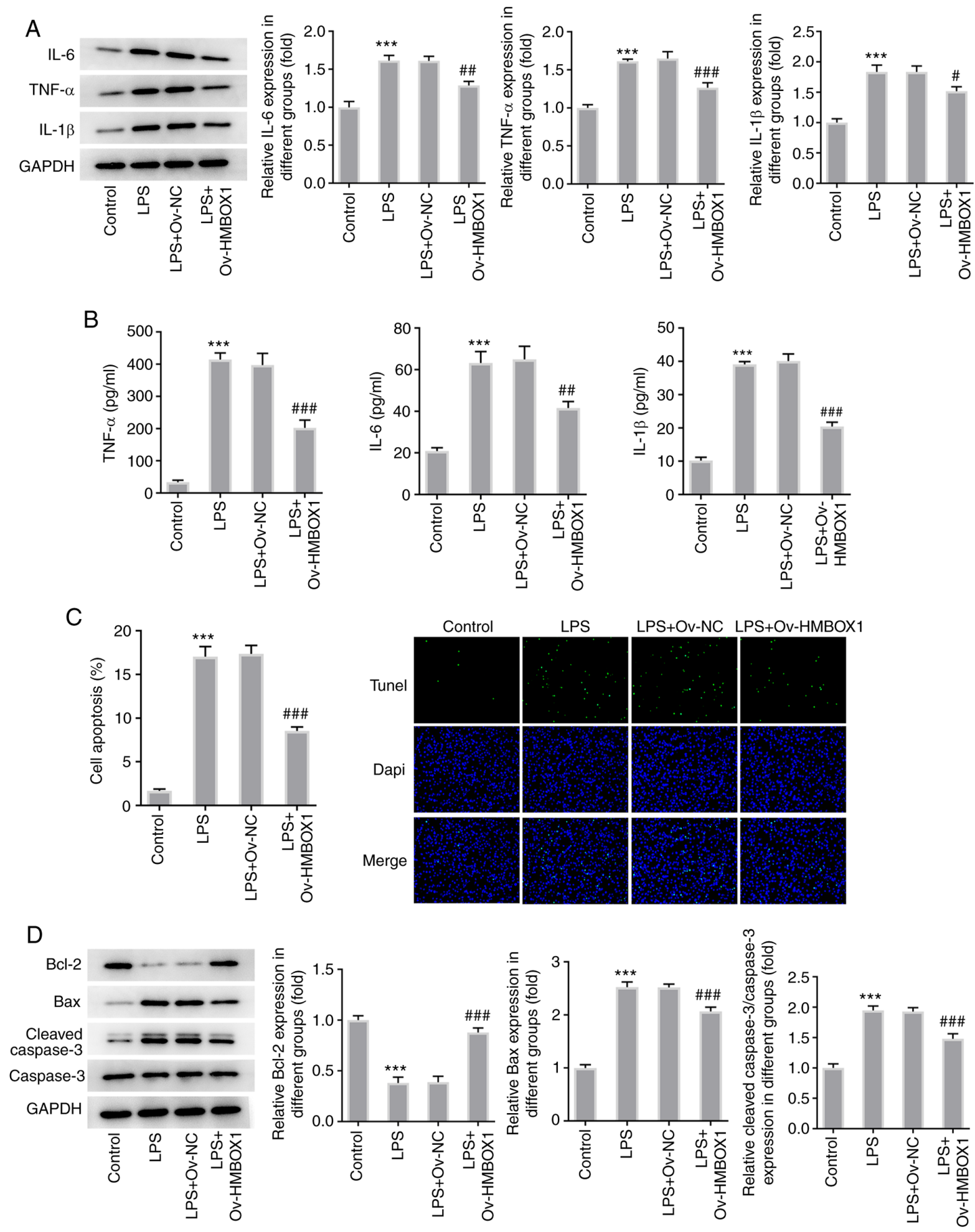

Figure 3. Overexpression of HMBOX1 blunts the inflammation and apoptosis of LPS-induced hPDLSCs. (A) Expression of inflammation-related proteins IL-6, TNF- $\alpha$ and IL-1 $\beta$ measured by western blotting. (B) Secretion of inflammation-related proteins IL-6, TNF- $\alpha$ and IL- $1 \beta$ measured by ELISA. (C) Apoptosis was measured and quantified by TUNEL. (D) Expression of apoptosis-related proteins Bcl-1, Bax and caspase 3 was measured by western blotting. ${ }^{* * *} \mathrm{P}<0.001$ vs. control; ${ }^{\# \mathrm{P}}<0.05,{ }^{\# \#} \mathrm{P}<0.01,{ }^{\# \# \#} \mathrm{P}<0.001$ vs. LPS+Ov-NC. hPDLSCs, human periodontal ligament stem cells; HMBOX1, Homeobox containing 1; LPS, lipopolysaccharide; Ov, overexpression; NC, negative control.

has been reported to be involved in cancer progression in an AKT-dependent manner, including osteosarcoma, lung cancer and liver cancer (23-25). However, whether MAPKs and AKT serve a role in the regulation of HMBOX1 upstream of inflammation and apoptosis in response to LPS require further study.
CXCL10 is an inflammatory cytokine that can be secreted by numerous cell types, including monocytes, endothelial cells, fibroblasts and mesenchymal cells (17). Previous studies reported that CXCL10 can regulate cell recruitment, migration and invasion $(26,27)$. This subsequently attracted 
A

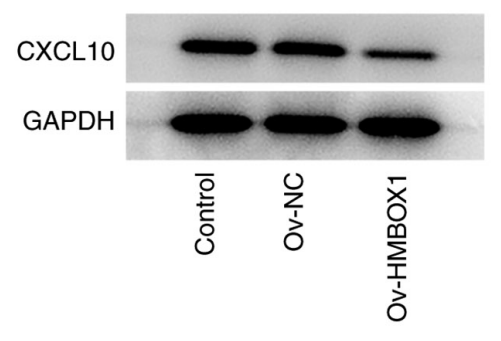

B

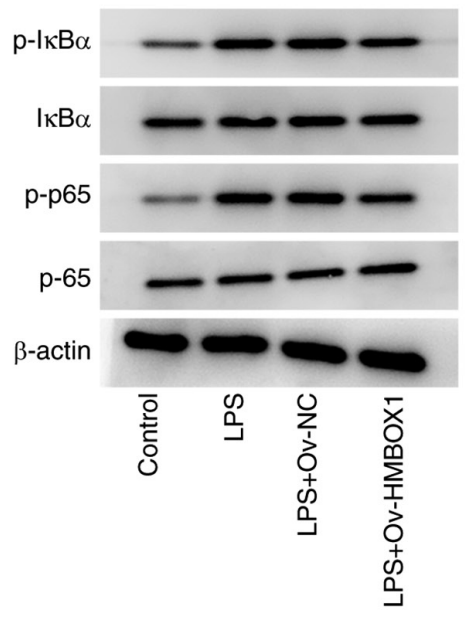

C

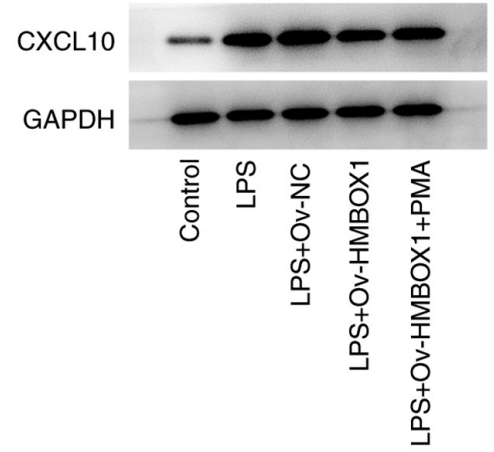

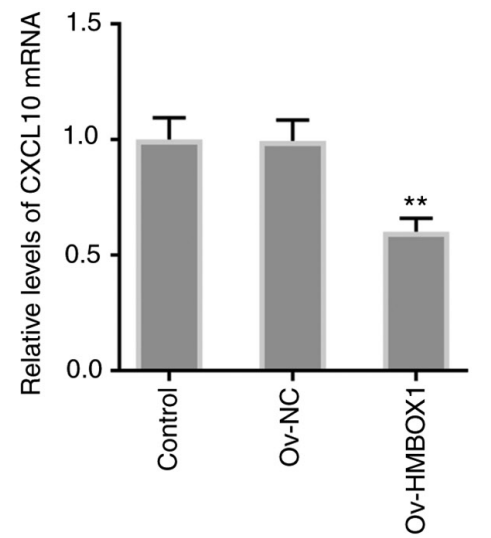
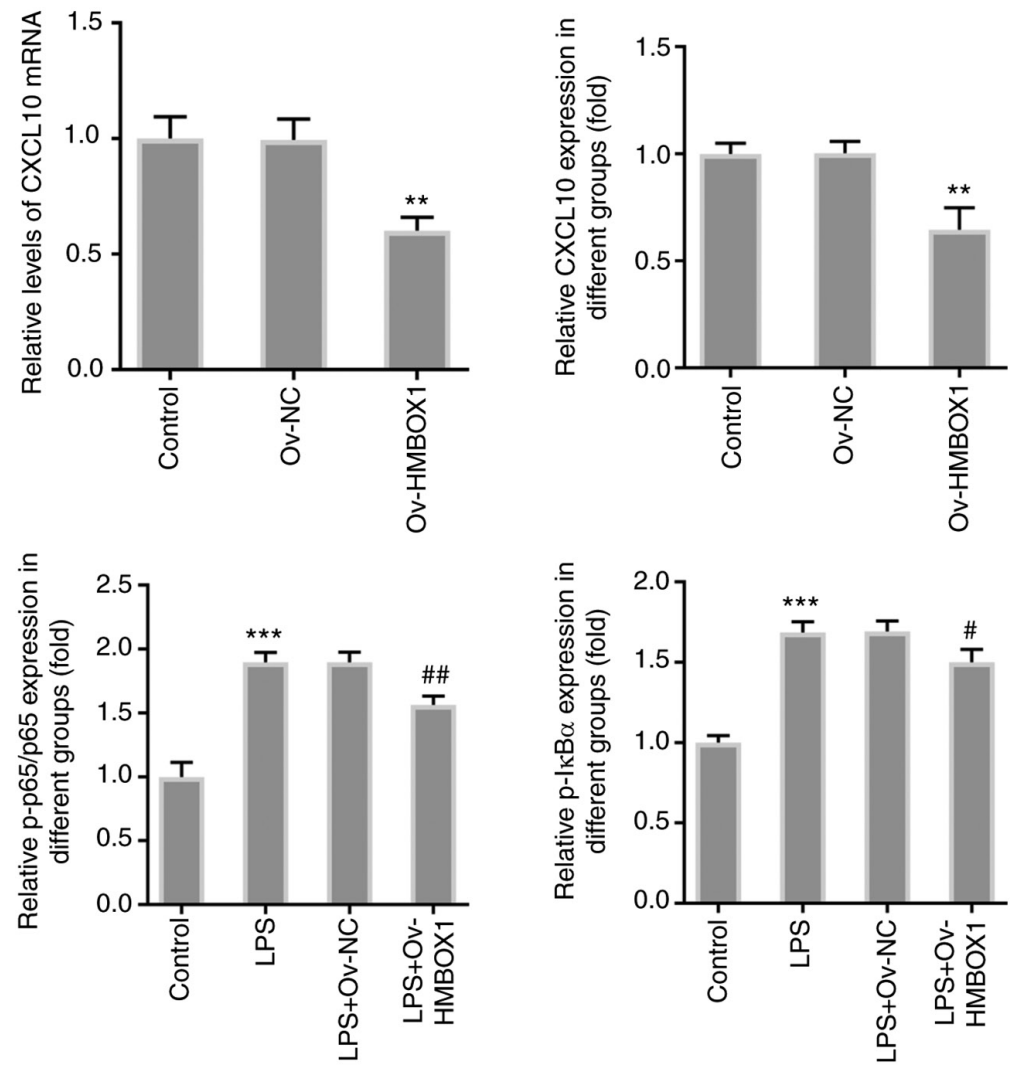

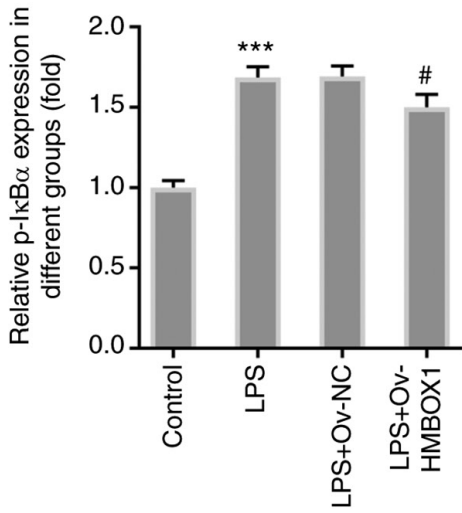

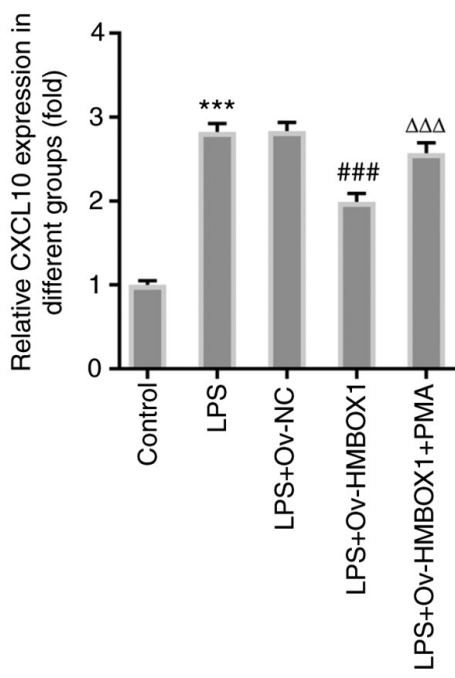

Figure 4. HMBOX1 modulates the expression of CXCL10 through the NF- $\kappa B$ signaling pathway. (A) The expression of CXCL10 in LPS-induced hPDLSCs overexpressing HMBOX1 was measured by western blotting and reverse transcription-quantitative PCR. (B) Phosphorylation of NF- $\mathrm{KB}$ signaling pathway-related proteins I $\mathrm{B} \alpha$ and $\mathrm{p} 65$ in LPS-induced hPDLSCs overexpressing HMBOX1 was measured by western blotting. (C) After PMA treatment, the expression of CXCL10 in LPS-induced hPDLSCs overexpressing CXCL10 was measured. ${ }^{* *} \mathrm{P}<0.01,{ }^{* * * *} \mathrm{P}<0.001$ vs. control; ${ }^{\#} \mathrm{P}<0.05,{ }^{\# \#} \mathrm{P}<0.01,{ }^{\# \# \#} \mathrm{P}<0.001$ vs. LPS+Ov-NC; ${ }^{\Delta \Lambda \Delta} \mathrm{P}<0.001$ vs. LPS+Ov-HMBOX1. hPDLSCs, human periodontal ligament stem cells; HMBOX1, Homeobox containing 1; LPS, lipopolysaccharide; Ov, overexpression; NC, negative control; CXCL10, C-X-C motif chemokine ligand 10; PMA, phorbol 12-myristate 13-acetate.

the attention of researchers, who later proposed that the expression profile of CXCL10 can be used as a novel type of biomarker for acute lung injury (28). CXCL10 was also discovered to serve a regulatory role during the inflammatory response against hepatic ischemia and reperfusion injury, suggesting its potential for use in a novel therapeutic approach for this disease (29). In addition, a previous study reported a close association between CXCL10 expression and periodontitis (30).
The NF- $\mathrm{KB}$ signaling pathway is an extensively studied signaling pathway that can modulate the secretion of cytokines, chemokines and adhesion molecules (20). Activation of the NF- $\mathrm{KB}$ signaling pathway by osteopontin was found to increase bone destruction during periapical periodontitis (31). In addition, another study reported that NF- $\mathrm{KB}$ serve an important role in the pathogenesis of periodontitis (32). It was also previously reported that $\mathrm{NF}-\kappa \mathrm{B}$ signaling was activated in highly inflamed white 

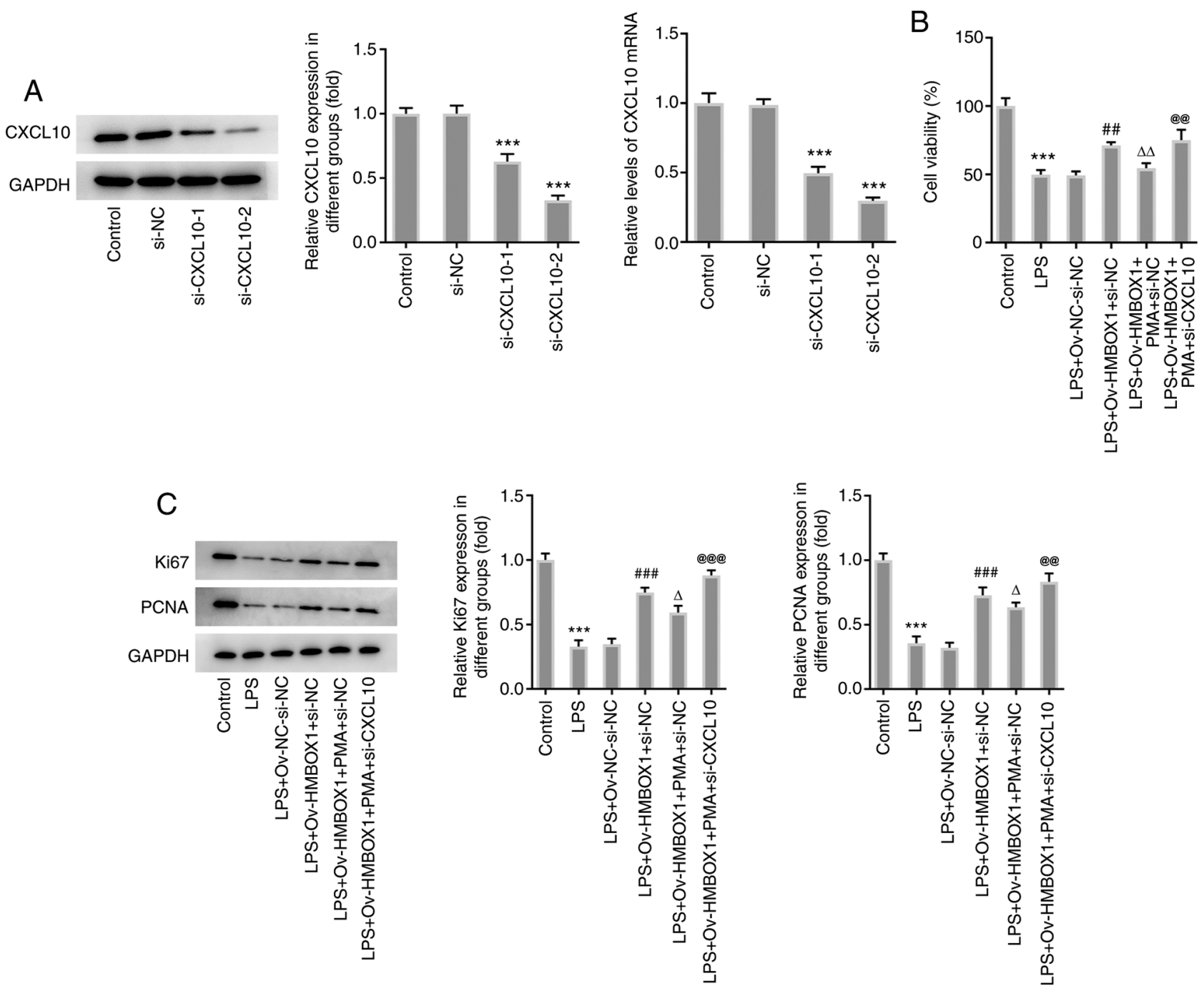

Figure 5. HMBOX1 enhances the proliferation of LPS-induced hPDLSCs through the NF- $\kappa B / C X C L 10$ axis. (A) The expression level of CXCL10 after knocking down its expression by si-CXCL10-1 and si-CXCL10-2 was measured by western blotting and reverse transcription-quantitative PCR. After PMA treatment, (B) cell viability and (C) the expression of proliferation-related proteins Ki-67 and PCNA in LPS-induced hPDLSCs co-transfected with Ov-HMBOX1 and

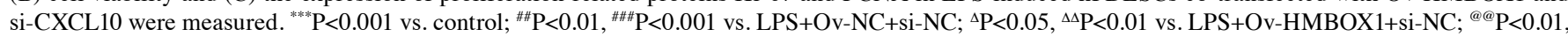
${ }^{\circledR @} \mathrm{P}<0.001$ vs. LPS+Ov-HMBOX1+PMA+si-NC. hPDLSCs, human periodontal ligament stem cells; HMBOX1, Homeobox containing 1; LPS, lipopolysaccharide; Ov, overexpression; NC, negative control; CXCL10, C-X-C motif chemokine ligand 10; PMA, phorbol 12-myristate 13-acetate; PCNA, proliferating cell nuclear antigen; si, small interfering.

adipose tissues in obese rats with periodontitis compared with that in obese rats without periodontitis, suggesting a role for NF- $\mathrm{\kappa B}$ in the pathogenesis of periodontitis (33). In the present study, following the treatment of PMA, an activator of the NF- $\mathrm{kB}$ signaling pathway, CXCL10 expression were reduced. In addition, hPDLSCs treated with PMA showed decreased cell viability and increased apoptosis, and increased levels of proinflammatory factors TNF- $\alpha$, IL- 6 and IL- $1 \beta$ compared with those in cells treated with LPS and transfected with Ov-HMBOX1 but without PMA treatment. However, the effects of inhibiting the NF- $\mathrm{KB}$ signaling pathway on CXCL10 expression require further study, which is a limitation of the present study. The NF- $\mathrm{BB}$ signaling pathway has also been implicated in the regulation of CXCL10. For example, the modulation of NF- $\mathrm{kB}$ was found to be mediated by COMM domain-containing 7 during anti-hepatocellular carcinoma therapy via CXCL10 upregulation (18). Furthermore, NF- $\kappa \mathrm{B}$ signaling was revealed to regulate CXCL10 production in $4 \mathrm{~T} 1$ breast cancer cells (34). Findings of the present study revealed that LPS-induced upregulation of CXCL10 expression was downregulated by Ov-HMBOX1 transfection, which was subsequently reversed by PMA treatment. These results support the hypothesis that HMBOX1 may regulate the physiology of LPS-induced hPDLSCs through the NF- $\mathrm{BB} / \mathrm{CXCL} 10$ signaling axis. Furthermore, CXCL10 knockdown reversed the PMA-induced inhibition of cell proliferation whilst reducing the PMA-induced production of inflammatory cytokines and apoptosis in these cells.

In conclusion, to the best of our knowledge, the present study is the first to provide evidence to suggest that HMBOX1 overexpression can attenuate LPS-induced hPDLSC injury by downregulating CXCL10 expression through the NF- $\mathrm{KB}$ signaling pathway. This may provide a novel insight into the development of targeted treatment options for periodontitis in the future. 
A

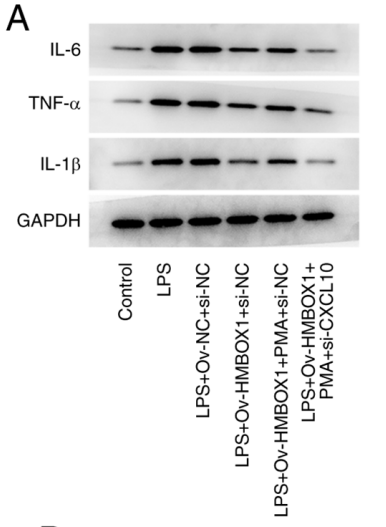

B

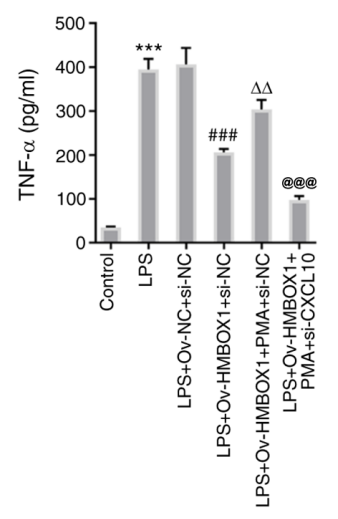

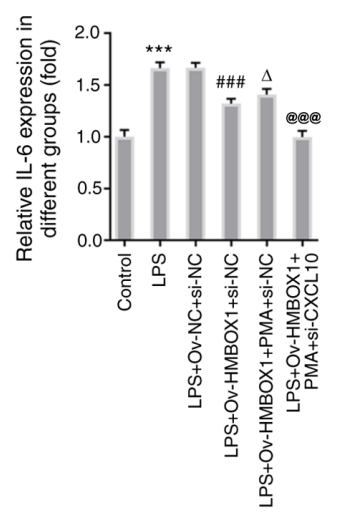

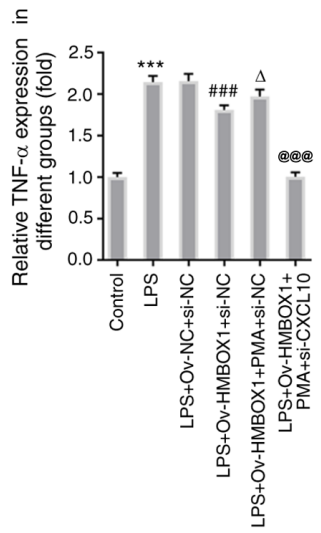

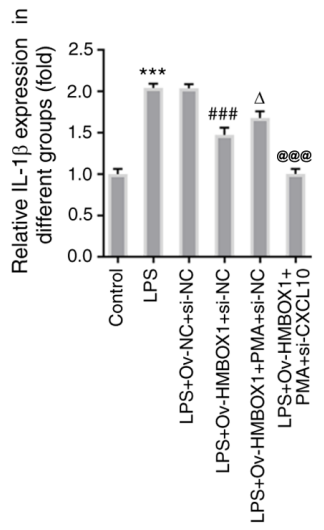

C
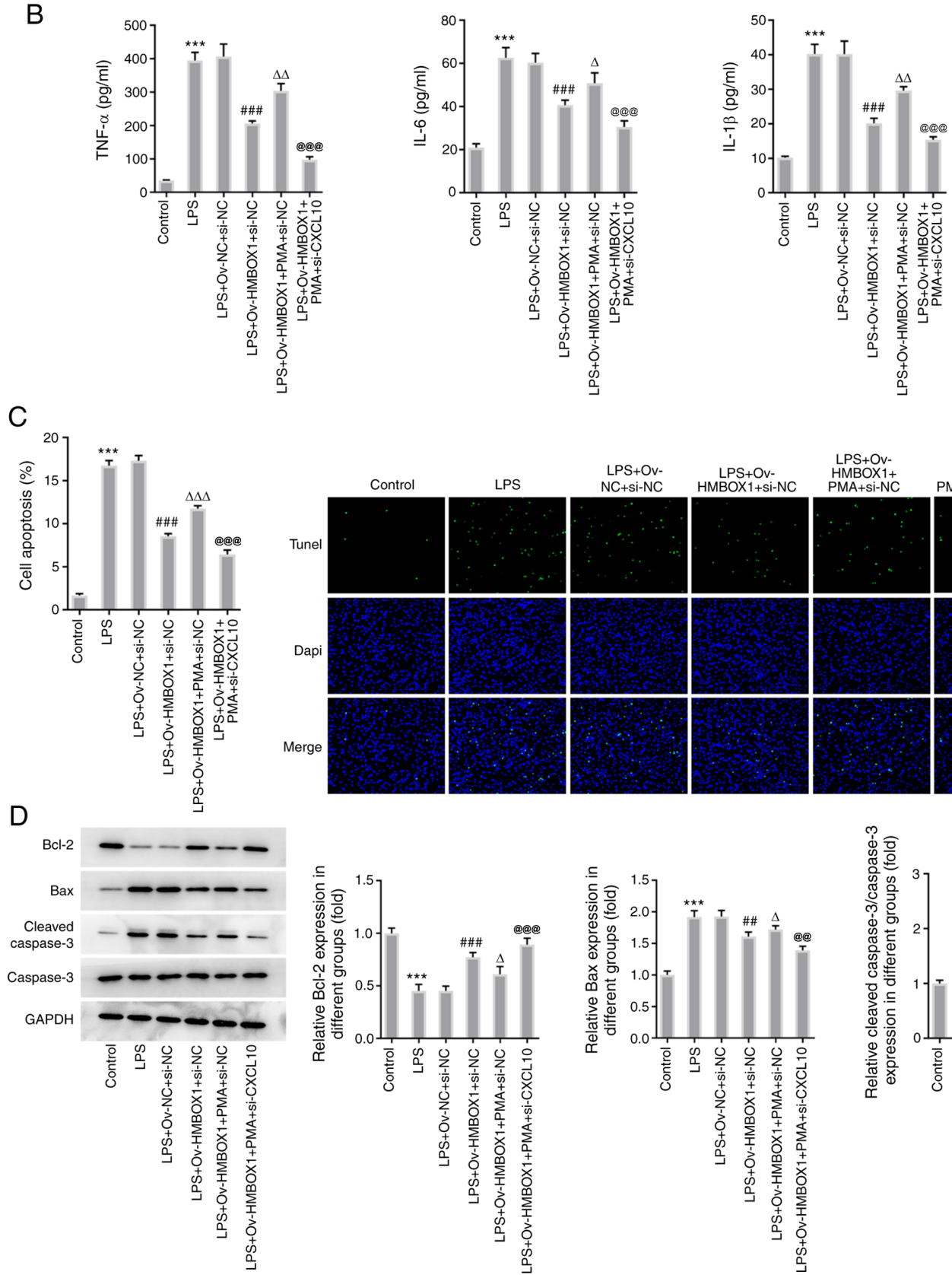

LPS+OV-
HMBOX1

$\mathrm{HMBOX} 1+$
$\mathrm{PMA+Si-NC}$

$\mathrm{LPS}+\mathrm{OV}-$
$\mathrm{HMBOX} 1+$

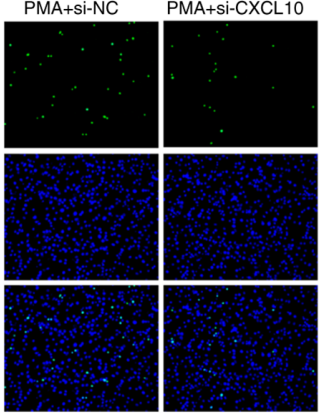

D
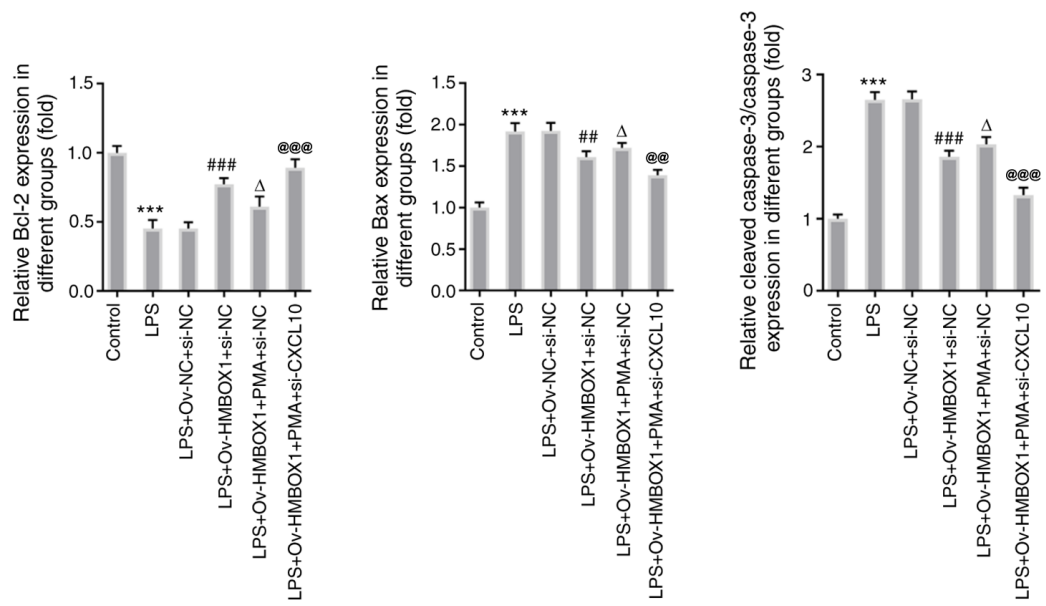

Figure 6. HMBOX1 blunts the inflammation and apoptosis of LPS-induced hPDLSCs through the NF- $\kappa$ B/CXCL10 axis. After PMA treatment, (A) the expression of inflammatory factors IL- 6 , TNF- $\alpha$ and IL- $1 \beta$ was measured by western blotting, whilst (B) the secretion of inflammatory factors IL-6, TNF- $\alpha$ and IL- $1 \beta$ was measured by ELISA. After PMA treatment, (C) apoptosis was measured using TUNEL, whereas (D) the expression of apoptosis-related proteins Bcl-2, Bax and caspase 3 in LPS-induced hPDLSCs transfected with Ov-HMBOX1 and si-CXCL10 was measured by western blotting. ${ }^{* * *} \mathrm{P}<0.001$ vs. control; ${ }^{\# \#} \mathrm{P}<0.01$,

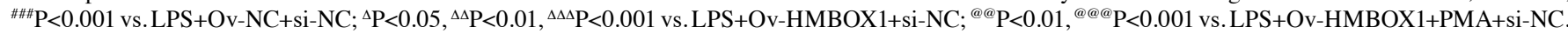
hPDLSCs, human periodontal ligament stem cells; HMBOX1, Homeobox containing 1; LPS, lipopolysaccharide; Ov, overexpression; NC, negative control; CXCL10, C-X-C motif chemokine ligand 10; PMA, phorbol 12-myristate 13-acetate; si, small interfering. 


\section{Acknowledgements}

No applicable.

\section{Funding}

No funding was received.

\section{Availability of data and materials}

The datasets used and/or analyzed during the current study are available from the corresponding author on reasonable request.

\section{Authors' contributions}

MN, HL, PL and PD conceived and designed the study, performed the experiment, collected, analyzed and interpreted the data and revised the manuscript. $\mathrm{MN}$ wrote the manuscript. All authors read and approved the final manuscript. MN and PD confirm the authenticity of all the raw data.

\section{Ethics approval and consent to participate}

Not applicable.

\section{Patient consent for publication}

Not applicable.

\section{Competing interests}

The authors declare that they have no competing interests.

\section{References}

1. Krishna R and De Stefano JA: Ultrasonic vs. hand instrumentation in periodontal therapy: Clinical outcomes. Periodontol 2000 71: 113-127, 2016.

2. Van Dyke TE: The management of inflammation in periodontal disease. J Periodontol 79 (Suppl 8): S1601-S1608, 2008.

3. Papapanou PN, Sanz M, Buduneli N, Dietrich T, Feres M, Fine DH, Flemmig TF, Garcia R, Giannobile WV, Graziani F, et al: Periodontitis: Consensus report of workgroup 2 of the 2017 World Workshop on the classification of periodontal and Peri-implant diseases and conditions. J Periodontol 89 (Suppl 1): S173-S182, 2018.

4. Slots J: Periodontitis: Facts, fallacies and the future. Periodontol 2000 75: 7-23, 2017.

5. Borgnakke WS, Ylostalo PV, Taylor GW and Genco RJ: Effect of periodontal disease on diabetes: Systematic review of epidemiologic observational evidence. J Periodontol 40 (Suppl 14): S135-S152, 2013.

6. Manresa C, Sanz-Miralles EC, Twigg J and Bravo M: Supportive periodontal therapy (SPT) for maintaining the dentition in adults treated for periodontitis. Cochrane Database Syst Rev 1: CD009376, 2018.

7. Chen S, Saiyin H, Zeng X, Xi J, Liu X, Li X and Yu L: Isolation and functional analysis of human HMBOX1, a homeobox containing protein with transcriptional repressor activity. Cytogenet Genome Res 114: 131-136, 2006.

8. Diao N, Li Y, Yang J, Jin C, Meng X, Jiao W, Feng J, Liu Z and Lu N: High expression of HMBOX1 contributes to poor prognosis of gastric cancer by promoting cell proliferation and migration. Biomed Pharmacother 115: 108867, 2019.
9. Yuan HX, Feng XE, Liu EL, Ge R, Zhang YL, Xiao BG and Li QS: 5,2'-dibromo-2,4',5'-trihydroxydiphenylmethanone attenuates LPS-induced inflammation and ROS production in EA.hy926 cells via HMBOX1 induction. J Cell Mol Med 23: 453-463, 2019.

10. Ma H, Su L, He X and Miao J: Loss of HMBOX1 promotes LPS-induced apoptosis and inhibits LPS-induced autophagy of vascular endothelial cells in mouse. Apoptosis 24: 946-957, 2019.

11. Guzeldemir-Akcakanat E, Alkan B, Sunnetci-Akkoyunlu D, Gurel B, Balta VM, Kan B, Akgun E, Yilmaz EB, Baykal AT, Cine N, et al: Molecular signatures of chronic periodontitis in gingiva: A genomic and proteomic analysis. J Periodontol 90: 663-673, 2019.

12. Zhao H, Han Q, Lu N, Xu D, Tian Z and Zhang J: HMBOX1 in hepatocytes attenuates LPS/D-GalN-induced liver injury by inhibiting macrophage infiltration and activation. Mol Immunol 101: 303-311, 2018.

13. Zhang F, Mears JR, Shakib L, Beynor JI, Shanaj S, Korsunsky I, Nathan A, Accelerating Medicines Partnership Rheumatoid Arthritis and Systemic Lupus Erythematosus (AMP RA/SLE) Consortium, Donlin LT and Raychaudhuri S: IFN- $\gamma$ and TNF- $\alpha$ drive a CXCL10 ${ }^{+}$CCL2 ${ }^{+}$macrophage phenotype expanded in severe COVID-19 and other diseases with tissue inflammation. bioRxiv: Aug 5, 2020 (Epub ahead of print). doi: 10.1101/2020.08.05.238360.

14. Luster AD, Unkeless JC and Ravetch JV: Gamma-interferon transcriptionally regulates an early-response gene containing homology to platelet proteins. Nature 315: 672-676, 1985.

15. Groom JR and Luster AD: CXCR3 ligands: Redundant, collaborative and antagonistic functions. Immunol Cell Biol 89: 207-215, 2011.

16. Gao J, Wu L, Wang S and Chen X: Role of chemokine (C-X-C Motif) ligand $10(\mathrm{CXCl10})$ in renal diseases. Mediators Inflamm 2020: 6194864, 2020.

17. Aldahlawi S, Youssef AR and Shahabuddin S: Evaluation of chemokine CXCL10 in human gingival crevicular fluid, saliva, and serum as periodontitis biomarker. J Inflamm Res 11: 389-396, 2018.

18. You N, Li J, Huang X, Wu K, Tang Y, Wang L, Li H, Mi N and Zheng L: COMMD7 activates CXCl10 production by regulating $\mathrm{NF}-\kappa \mathrm{B}$ and the production of reactive oxygen species. Mol Med Rep 17: 6784-6788, 2018

19. Chen H, Lin W, Lin P, Zheng M, Lai Y, Chen M, Zhang Y, Chen J, Lin X, Lin L, et al: IL-10 produces a dual effect on OGD-induced neuronal apoptosis of cultured cortical neurons via the NF- $\mathrm{BB}$ pathway. Aging (Albany NY) 11: 10796-10813, 2019.

20. Livak KJ and Schmittgen TD: Analysis of relative gene expression data using real-time quantitative PCR and the 2(-Delta Delta C(T)) method. Methods 25: 402-408, 2001.

21. Zhou W, Su L, Duan X, Chen X, Hays A, Upadhyayula S, Shivde J, Wang H, Li Y, Huang D and Liang S: MicroRNA-21 down-regulates inflammation and inhibits periodontitis. Mol Immunol 101: 608-614, 2018

22. Holland PW, Booth HA and Bruford EA: Classification and nomenclature of all human homeobox genes. BMC Biol 5: 47, 2007.

23. Chen S, Li Y, Zhi S, Ding Z, Wang W, Peng Y, Huang Y, Zheng R, Yu H, Wang J, et al: WTAP promotes osteosarcoma tumorigenesis by repressing $\mathrm{HMBOX} 1$ expression in an $\mathrm{m}^{6} \mathrm{~A}$-dependent manner. Cell Death Dis 11: 659, 2020.

24. Li J, Zhao Y and Wang J: Extracellular vesicle-associated microRNA-221-3p secreted by drug-resistant lung cancer cells targets HMBOX1 to promote the progression of lung cancer. Cancer Gene Ther 28: 679-692, 2021.

25. Zhao H, Jia H, Han Q and Zhang J: Homeobox containing 1 inhibits liver cancer progression by promoting autophagy as well as inhibiting stemness and immune escape. Oncol Rep 40: 1657-1665, 2018.

26. Tsai CF, Chen JH and Yeh WL: Pulmonary fibroblasts-secreted CXCL10 polarizes alveolar macrophages under pro-inflammatory stimuli. Toxicol Appl Pharmacol 380: 114698, 2019.

27. McGrath-Morrow SA, Lee S, Gibbs K, Lopez A, Collaco JM, Neptune E, Soloski MJ, Scott A and D'Alessio F: Immune response to intrapharyngeal LPS in neonatal and juvenile mice. Am J Respir Cell Mol Biol 52: 323-331, 2015.

28. Chalin A, Lefevre B, Devisme C, Pronier C, Carrière V, Thibault V, Amiot L and Samson M: Serum CXCL10, CXCL11, CXCL12, and CXCL14 chemokine patterns in patients with acute liver injury. Cytokine 111: 500-504, 2018 
29. Zhai Y, Shen XD, Gao F, Zhao A, Freitas MC, Lassman C, Luster AD, Busuttil RW and Kupiec-Weglinski JW: CXCL10 regulates liver innate immune response against ischemia and reperfusion injury. Hepatology 47: 207-214, 2008.

30. Shimada Y, Tabeta K, Sugita N and Yoshie H: Profiling biomarkers in gingival crevicular fluid using multiplex bead immunoassay. Arch Oral Biol 58: 724-730, 2013.

31. Dong M, Yu X, Chen W, Guo Z, Sui L, Xu Y, Shang Y, Niu W and Kong Y: Osteopontin promotes bone destruction in periapical periodontitis by activating the NF- $\kappa$ B pathway. Cell Physiol Biochem 49: 884-898, 2018.

32. Hiyari S, Wong RL, Yaghsezian A, Naghibi A, Tetradis S, Camargo PM and Pirih FQ: Ligature-induced peri-implantitis and periodontitis in mice. J Clin Periodontol 45: 89-99, 2018.
33. Huang Y, Zeng J, Chen G, Xie X, Guo W and Tian W: Periodontitis contributes to adipose tissue inflammation through the NF-B, JNK and ERK pathways to promote insulin resistance in a rat model. Microbes Infect 18: 804-812, 2016.

34. Jin WJ, Kim B, Kim D, Park Choo HY, Kim HH, Ha H and Lee $\mathrm{ZH}$ : NF- $\mathrm{KB}$ signaling regulates cell-autonomous regulation of CXCL10 in breast cancer 4T1 cells. Exp Mol Med 49: e295, 2017.

cc) (i) $(9$ This work is licensed under a Creative Commons Attribution-NonCommercial-NoDerivatives 4.0 International (CC BY-NC-ND 4.0) License. 